\title{
Bilingüismo y Psicoterapia
}

\author{
Ana Lía Laguzzi ${ }^{1}$
}

\section{Artículo}

Material original autorizado para la publicación en la revista Psicodebate. Facultad de Ciencias Sociales. Universidad de Palermo.

Recibido 9-12-2013 | Aceptado 17-02-2014

\section{Resumen}

La literatura sobre el proceso psicoterapéutico en individuos bilingües sugiere que el bilingüismo de una persona puede afectar el proceso y/o el resultado del mismo. El incremento de la migración mundial ha dado como consecuencia la presencia de miles de individuos en países donde no se habla su lengua nativa y considerando que muchos de ellos pueden llegar a necesitar servicios psicológicos, este artículo explora ciertos fenómenos asociados con el bilingüismo que tienen importantes implicaciones para la tarea psicoterapéutica. Nuestro objetivo es establecer con claridad las razones por las cuales consideramos de gran importancia que el terapeuta trabajando con pacientes bilingües esté familiarizado con dichos fenómenos y con su posible influencia en la evaluación diagnóstica y en el tratamiento de los mismos. Además, deseamos contribuir a ampliar la exploración de la influencia del bilingüismo en psicoterapia, formulando algunas preguntas de posible consideración en futuros estudios sobre este tema.

Palabras Clave: Migración - Bilingüismo - Fenómenos - Psicoterapia.

1 Buenos Aires, allaguzzi@hotmail.com 


\section{Abstract}

The literature on the psychotherapeutic process of bilingual individuals suggests that a person's bilingualism may affect the process and/or outcome of his/her psychotherapy. The increase in population migration all over the world has resulted in the presence of thousands of persons in countries where their language of origin is not spoken. Taking into consideration that many of those individuals might require psychological services, this article explores several bilingualismrelated phenomena that have important implications for the psychotherapeutic task. Our objective is to establish clearly the reasons why we consider of extreme importance that the therapist working with bilingual people be familiar with these phenomena and with their possible influence on their diagnostic evaluation and treatment. Furthermore, we would like to contribute to a larger exploration of bilingualism's influence on the psychotherapeutic process by formulating some questions for possible consideration in future studies on the subject.

Keywords: Migration - Bilingualism - Phenomena - Psychotherapy. 


\section{Fenómenos relacionados con el bilingüismo y su influencia en los procesos de evaluación y tratamiento psicológicos}

En las últimas décadas se ha registrado un flujo migratorio constante de aproximadamente el 3\% de la población mundial desde los países de origen hacia otras naciones. En Latinoamérica el 19.7\% de dicho desplazamiento ha sido en dirección a Estados Unidos y Canadá y el 3.1\% en dirección a Europa. Otro importante flujo migratorio ha desplazado al $15.7 \%$ y al 9.6\% de la población de Asia hacia Europa y hacia América del Norte, respectivamente. Los europeos han emigrado hacia Estados Unidos y Canadá (8.2\%) y Asia (8.6\%). El desplazamiento desde África hacia Europa ha sido del 7.2\% de su población. Las corrientes migratorias citadas son las de mayor envergadura, pero debe mencionarse la existencia de otras de menor volumen que abarcan no solamente a las Américas, Europa y Asia sino también a Oceanía (United Nations Development Programme, 2009).

Esto ha resultado en la presencia de miles de individuos en países donde la lengua dominante no es su lengua nativa. El fenómeno del bilingüismo ha sido extensivamente estudiado por lingüistas, educadores, sociólogos y psicólogos, pero la influencia del bilingüismo en los procesos de psicoterapia o de evaluación psicológica ha recibido poca atención. La limitada literatura existente sugiere que el bilingüismo de una persona puede afectar el proceso y/o el resultado de su psicoterapia. (Javier, 1989).

Las experiencias emocionales de una persona durante el período de adquisición de una lengua son afectadas por la edad y el desarrollo cognitivo del individuo, así como por el contexto en que dicha adquisición tiene lugar. La lengua "madre" se aprende muy temprano en la vida y en el ámbito familiar. Algunas veces el idioma hablado en el hogar es diferente del hablado en el amplio contexto social que lo rodea. Ocasionalmente, el lenguaje hablado por la madre o por la persona responsable de la crianza del niño es diferente al de otros miembros de la familia. Trabajos de investigación sobre bilingüismo apoyan la hipótesis de que el niño criado en un hogar bilingüe o en un contexto bilingüe tendrá diferentes experiencias emocionales asociadas con cada lenguaje (Ervin, 1964; Kolers, 1968; Lambert, 1956; Laguzzi, 1989; Kokaliari \& Catanzarite, 2011).

Si ser expuesto a idiomas diferentes durante la infancia puede tener implicaciones emocionales para un individuo, surge la pregunta sobre la posible existencia de emociones vinculadas a la adquisición de un segundo lenguaje en la vida adulta. Cuando un adulto empieza a aprender y a usar un nuevo idioma, con frecuencia se encuentra esforzándose por comunicar sus pensamientos de la misma manera en que un niño pequeño lo hace cuando comienza a hablar. Pero mientras un niño, como lo señala Stengel (1939) "no teme decir tonterías, porque hacerlo le 
produce placer" (p. 478) la mayoría de los adultos aprendiendo un nuevo lenguaje sienten ese temor. Por lo tanto, la adquisición de un nuevo lenguaje coloca a un adulto en una situación que puede lastimarle narcisísticamente y resultarle difícil de tolerar. Además, si tal adquisición tiene lugar en el contexto de una experiencia totalmente diferente y potencialmente traumática en su vida, como ser la emigración, es razonable especular que el uso del segundo lenguaje en la vida adulta tendrá implicaciones emocionales y que las mismas muy probablemente serán diferentes a aquellas asociadas con el uso de la lengua madre.

La trayectoria profesional de esta autora tuvo lugar durante casi 20 años en los Estados Unidos y específicamente en la ciudad de Nueva York. En los hospitales y clínicas de dicha ciudad una considerable proporción de la población que es evaluada psiquiátricamente y/o está involucrada en psicoterapia es bilingüe, predominantemente hispano-parlante. Una experiencia surgida durante la psicoterapia de una adolescente bilingüe despertó mi interés en el rol del bilingüismo en psicoterapia.

La paciente había nacido en los Estados Unidos de padres hispano-parlantes. Había estado expuesta a ambos idiomas desde su infancia pero consideraba al inglés como su idioma dominante. La terapia era siempre conducida en inglés. Un día la paciente contó un sueño en el que había experimentado un miedo intenso de naturaleza poco clara. Asociar libremente no produjo nada significativo. Pregunté a la joven si alguna vez había experimentado un miedo parecido y ella insistió enfáticamente en que "era un miedo diferente al que hubiera sentido en cualquier otro momento de su vida".

El sueño preocupaba a la paciente y lo mencionó repetidamente durante sesiones posteriores, pero nos fue imposible lograr alguna comprensión del mismo. Un día, cuando lo mencionó una vez más, le pregunté otra vez: “¿Nunca antes experimentaste un miedo parecido?" Pero esta vez le hice la pregunta en castellano (mi uso de ese idioma fue un "accidente"). En ese momento una extraña expresión apareció en su rostro y sus ojos parecían fuera de foco. Después de unos minutos de silencio, la joven empezó a contarme, en español, la historia de un incidente durante el cual ella casi se había ahogado en la bañera. Esto había ocurrido cuando ella tenía 3 años de edad y no lo había recordado nunca hasta ese momento. En mi opinión, la paciente pudo recobrar esa memoria cuando, involuntariamente, yo le había dado una pista o clave al usar el lenguaje en el cual la memoria había sido guardada. A los 3 años, la niña sólo hablaba español.

La experiencia que acabo de describir se vincula a algunos de los fenómenos reportados en la literatura clínica y a las conclusiones de estudios de investigación sobre bilingüismo y psicoterapia (Schrauf, 2000; Schrauf \& Rubin, 2000; Burck, 2004). Los principales fenómenos identificados son: la barrera del lenguaje, la 
independencia del lenguaje, y el autoconcepto. El primero se refiere a aquellos individuos cuyo dominio del segundo idioma es muy inferior al de su lengua nativa (bilingües subordinados). Los otros dos están vinculados con aquellas personas que son muy competentes en ambos lenguajes (bilingües compuestos y coordinados).

La barrera del lenguaje es el grado de deficiencia lingüística exhibido por bilingües subordinados lo que resulta en que experimenten dificultades al intentar comunicarse en su segundo lenguaje. En la psicoterapia de estos individuos ésta se puede manifestar en: a) el uso de ciertos mecanismos de defensa y b) la ausencia de integración entre experiencia y afecto. La explicación psicoanalítica de este efecto es que las palabras de un segundo lenguaje tienen menor peso emocional porque fueron adquiridas durante experiencias no tan cruciales para la formación de la personalidad como las relacionadas con la adquisición de la lengua madre. Otro punto de vista explica dicho distanciamiento emocional como producto del proceso por el cual las ideas, sentimientos, etc., de una persona son expresados en un determinado lenguaje. Este proceso depende de la cantidad de palabras en ese lenguaje de las cuales dispone el individuo (factor léxico), su capacidad para ordenarlas gramaticalmente (factor sintáctico) y su capacidad para pronunciarlas (factor fonético). El uso del segundo lenguaje obliga además a estas personas a la constante operación mental de traducir del y al lenguaje dominante. Esto le impone exigencias cognitivas adicionales que pueden causar un desplazamiento de energía afectiva: la persona invierte afecto en cómo dice las cosas, en vez de en lo que dice (Marcos, 1976), facilitando así el uso de ciertas defensas, particularmente en pacientes con rasgos de personalidad obsesivos. Estas personas suelen preocuparse en exceso por el léxico y la gramática de su discurso en el segundo lenguaje, lo que con frecuencia resulta en intelectualización excesiva y en expresión verbal emocionalmente estéril.

Resumiendo, la barrera del lenguaje puede representar un obstáculo en psicoterapia al interferir con la integración de experiencia y afecto por parte del paciente o facilitando su uso de defensas obsesivas (Marcos, 1976, 1979; Marcos, Urcuyo, Kesselman, \& Alpert, 1973; Kokaliari \& Catanzarite, 2011). Sin embargo, por otra parte, dicha barrera puede, en algunos casos, facilitar el acceso del paciente a material emocionalmente tan intenso que le provoca excesiva ansiedad en su idioma nativo (Krapf, 1955; Caruso, citado por Marcos, 1976; Costa \& Dewaele, 2012).

El fenómeno de la independencia del lenguaje se refiere a la capacidad que poseen algunos individuos perfectamente bilingües de mantener y operar dos sistemas de lenguaje separados. La evidencia de la existencia de dicho fenómeno proviene de la neurología, la psicolingüística y el psicoanálisis. Estudios neurológicos de afasia en personas bilingües han demostrado que luego de accidentes cerebrovasculares o durante síndromes de deterioro cerebral, estos 
individuos exhiben déficits diferentes en sus dos idiomas (Lipsius, 1975; Albert \& Obler, 1978). Hay diferentes interpretaciones de este fenómeno; la hipótesis sugerida por algunos neurólogos es la de la existencia de un área anatómica en el cerebro específicamente involucrada en cada idioma. Sea cual sea la razón, la mayoría de los investigadores concuerdan en que los mecanismos asociados con el uso del lenguaje primario son más resistentes al deterioro orgánico que aquellos correspondientes al lenguaje secundario (Marcos \& Alpert, 1976).

Estudios de psicolingüística han demostrado empíricamente que individuos bilingües tienen asociaciones diferentes en respuesta a palabras semánticamente idénticas según el lenguaje que se use (Lenneberg \& Roberts, 1956). Palabras que se refieren a sentimientos evocan las respuestas menos parecidas (Kolers, 1968). Un estudio que comparaba las respuestas de competentes bilingües en inglés y francés en el Test de Percepción Temática (TAT) reveló marcadas diferencias en las historias contadas y las emociones expresadas según el lenguaje en que el individuo fue evaluado (Ervin, 1964). La literatura psicoanalítica ofrece numerosos ejemplos de la existencia de zonas del mundo intrapsíquico del paciente que permanecen ocultas porque son independientes del lenguaje en el que se conduce el tratamiento (Greenson, 1950; Marcos, 1972; Javier, 1989). Marcos y Alpert (1976) señalan que las relativas consecuencias de este fenómeno en el proceso terapéutico puede depender de la magnitud del mismo y de la relevancia del material oculto en relación a las metas del tratamiento.

Resumiendo, el fenómeno de la independencia del lenguaje ha sido identificado principalmente en competentes bilingües coordinados, es decir, personas que han adquirido sus dos lenguajes en diferentes contextos culturales y en diferentes etapas de su vida. Según hemos visto, en la psicoterapia de estas personas este fenómeno puede resultar en que material de importancia no sea accesible en uno de los idiomas (Buxbaum, 1949; Greenson, 1950; Marcos, 1980; Marcos \& Alpert, 1976; Kokaliari \& Catanzarite, 2011). Por lo tanto, algunas áreas del mundo psíquico de estos pacientes, por ejemplo, algunas memorias de la infancia, pueden permanecer inexploradas porque son independientes del sistema de lenguaje en el cual se realiza la psicoterapia. Otra posible manifestación de este fenómeno es la ausencia de contenido afectivo en la verbalización de pensamientos o experiencias cuya naturaleza sugiere una intensa carga emocional (Krapf, 1955; Marcos, 1972; Marcos \& Alpert, 1976; Harris, 2006).

Finalmente, el tercer fenómeno vinculado al bilingüismo que parece tener importantes implicaciones en psicoterapia es la experiencia, reportada por algunos pacientes, de poseer un diferente concepto de identidad personal en cada idioma (Marcos, Eisma, \& Guimon, 1977). Estos pacientes tienden a ser competentes bilingües coordinados que dicen sentirse como dos personas diferentes según el 
lenguaje que usen, o comportarse de una manera automática o compulsiva en uno de sus dos idiomas. Greenson relata el caso de una paciente bilingüe en alemán e inglés, que se describía como una persona diferente en cada lenguaje: "en alemán soy una niña asustada y sucia, en inglés soy una mujer nerviosa y refinada" (Greenson, 1950, p. 19). Estos informes clínicos parecen apoyar la teoría de la relatividad del lenguaje, también conocida como la hipótesis Sapir-Whorf. Esta polémica noción postula que el lenguaje no es solamente un vehículo para la comunicación, sino que el lenguaje mismo representa una realidad por la cual la gente percibe, estructura y organiza su mundo.

Los pacientes bilingües generalmente solicitan tratamiento por razones que no se refieren a conflictos de identidad relacionados con el lenguaje, pero con frecuencia este tipo de conflicto aparece cuando el tratamiento ya está bastante avanzado. En estos casos, éste se convierte en un importante elemento del mismo con ramificaciones conscientes e inconscientes (Marcos, Eisma, \& Guismón, 1977). A un nivel consciente, estos pacientes con frecuencia están deprimidos porque se sienten como dos personas diferentes o porque se dan cuenta de que actúan en una forma automática o compulsiva en uno de los lenguajes. Con frecuencia, aparecen deseos vinculados a cada lenguaje que pueden ser el resultado de los valores culturales conectados a ese idioma o que pueden estar asociados con los valores que el paciente atribuye a personas de importancia en su vida que hablan ese lenguaje.

Algunas veces los pacientes bilingües utilizan en forma inconsciente su doble sentido de identidad para negar aspectos inaceptables de su personalidad conectándolos a uno de sus dos idiomas. Este es con frecuencia el caso de los pacientes bilingües que tratan de negar la identidad vinculada a su lengua nativa, poniendo énfasis en una "nueva" identidad apoyada por su segundo lenguaje. En estos casos, el doble autoconcepto es con frecuencia el origen de conflicto y/o confusión y puede resultar en diferentes tipos de defensas (Marcos, 1980; Perez Foster, 1996; Pavlenko, 2006). Sin embargo, en algunos casos la identidad ligada al segundo idioma puede facilitar la exploración de problemas que son demasiado angustiantes para el paciente en su idioma primario.

\section{Conclusiones}

Los pacientes bilingües presentan problemas y oportunidades especiales para el terapeuta. En el caso de los bilingües subordinados, la barrera del lenguaje puede interferir con la integración de experiencia y afecto, facilitar el uso de mecanismos de defensa obsesivos y distorsionar la relación entre el paciente y el terapeuta. Sin embargo, algunas veces puede ser más fácil para el paciente acceder 
a material de gran intensidad emocional a través de su lenguaje no-dominante, ya que dicho material causaría excesiva ansiedad en su idioma primario.

En el tratamiento de bilingües coordinados el fenómeno de la independencia de lenguaje puede resultar en que material de importancia clínica no esté disponible en un idioma y en la experiencia de un doble concepto de identidad personal por parte de algunos pacientes.

En vista de todo lo anterior, es crucial que aquellos psicoterapeutas que traten a pacientes bilingües sean sensibles al significado e importancia de la experiencia bilingüe y estén familiarizados con las implicaciones de los fenómenos relacionados con la misma tanto durante la entrevista diagnóstica como durante el proceso de tratamiento.

Dos preguntas potencialmente interesantes surgen de nuestra discusión. La primera se refiere a la expresión afectiva del paciente en relación a su tipo de bilingüismo. Cabe señalar una vez más que la distinción entre bilingües coordinados y compuestos no radica en su nivel de competencia en cada idioma, ya que se asume que ambos grupos lo hablan con fluidez, sino en el contexto sociocultural en que ambos lenguajes han sido adquiridos. Bilingües compuestos los aprenden muy temprano en sus vidas y en el mismo contexto cultural y social, mientras que los coordinados adquieren su segundo idioma en un período posterior a la infancia y en un contexto cultural diferente. ¿Hay alguna diferencia en la capacidad de expresión afectiva según el idioma en uso entre estos dos tipos de personas bilingües? La mayoría de los estudios empíricos y de los casos clínicos reportados en la literatura involucran a bilingües coordinados y no abordan esta pregunta. Sin embargo, teniendo en cuenta que la lengua materna es la que se aprende al principio de la vida, es razonable presentar la noción que, aunque el individuo bilingüe compuesto no considere dicha lengua su idioma dominante o preferido, ésta será, salvo raras excepciones, la lengua de su madre. Por lo tanto, es posible postular la hipótesis de que la terapia conducida en el idioma de la madre puede facilitar en estos individuos la expresión de emociones o experiencias infantiles (Harris 2006; Laguzzi, 1989; Rosensky \& Gomez, 1983).

Las otras preguntas de interés conciernen al bilingüismo del terapeuta. ¿Es preferible que el paciente bilingüe se trate con un terapeuta igualmente bilingüe? ¿cuáles son las posibles ventajas y desventajas, si las hay? ¿influye en la alianza terapéutica el lenguaje usado en el tratamiento? ¿es la interacción del bilingüismo de ambos participantes significativa? ¿influye de algún modo la orientación teórica del terapeuta?

Las preguntas arriba presentadas sugieren posibles tópicos para trabajos de investigación en bilingüismo y psicoterapia. En la práctica clínica, es fundamental que tanto terapeutas bilingües como monolingües estén familiarizados con los 
fenómenos relacionados con el bilingüismo y su posible influencia en el tratamiento del paciente, y que sean sensibles a las manifestaciones derivadas de los mismos.

En nuestro mundo contemporáneo miles de individuos emigran de sus países de origen en busca de trabajo, asilo u oportunidades diversas. En consecuencia, muchas de estas personas se ven obligadas a buscar ayuda de distinta índole para sobrevivir en una cultura que les es extraña, incluyendo servicios de salud mental o de apoyo psicológico. Con frecuencia estos individuos no hablan el idioma del país en el cual se encuentran o lo hacen muy pobremente. Por lo tanto, en la sociedad actual donde el desplazamiento de miles de individuos a través de fronteras nacionales es un hecho cada vez más frecuente, la presencia en todo el mundo de terapeutas capacitados para el trabajo con clientes bilingües adquiere cada vez mayor urgencia. 


\section{Referencias}

Albert, M. \& Obler, L. (1978). The bilingual brain: Neuropsychological and neurological aspects of bilingualism. New York: Academic.

Burck, C. (2004). Living in several languages: implications for therapy. Journal of Family Therapy, 26, 314-339.

Buxbaum, E. (1949). The role of a second language in the formation of ego and superego. Psychoanalytic Quaterly, 18, 279-89.

Caruso, I. A., \& Duque, R. T. (1966). El problema de psicoanálisis en lengua extranjera. Archivos de Estudios Psicoanalíticos y Psicología Médica, 3, 7.

Costa, B. \& Dewaele, J. M. (2012). Psychotherapy across languages: beliefs, attitudes and practices of monolingual and multilingual therapists with their multilingual patients. Language and Psychoanalysis, 1, 19-41.

Ervin, S. (1964). Language and TAT content in bilinguals. Journal of Abnormal and Social Psychology, 68(5), 500-507.

Greenson, R. (1950). The mother tongue and the mother. International Journal of Psychoanalysis, 31, 18-23.

Harris, C. L. (2006). When is a first language more emotional? En A. Pavlenko (Ed.) Bilingual Minds: emotional experience, expression and representation (pp. 257-283). Clevedon, U.K.: Multilingual Matters.

Javier, R. A. (1989). Linguistic considerations in the treatment of bilinguals. Psychoanalytic Psychology, 6(1), 87-96.

Kokaliari, E. \& Catanzarite, G. (2011). Understanding the role of language in bilingual psychotherapy: clinical implications. Psychiatric Prax, 8, 363-413.

Kolers, P. A. (1968). Bilingualism and information processing. Scientific American, 218, 78-86.

Krapf, E. (1955). The choice of language in polyglot psychoanalysis. Psychoanalytic Quaterly, 24, 343-357.

Laguzzi, A. L. (1989). Effect of bilingualism on emotional content of free associations. Unpublished doctoral research project. New York: Ferkauf Graduate School of Psychology, Yeshiva University. 
Lambert, W. A. (1956). Developmental aspects of second language acquisition: Associational fluency, stimulus provocativeness and word order influence. Journal of Social Psychology, 43, 83.

Lennenberg, E. H. \& Roberts, J. M. (1956). The Language of Experience. Bloomington, Indiana: Indiana University Press.

Lipsius, L. H. (1975). Electroconvulsive therapy and language (letter to Ed.) American Journal of Psychiatry, 132, 459.

Marcos, L. R. (1972). Lying: a particular defense met in psychoanalytic therapy. American Journal of Psychoanalysis, 32, 195-202.

Marcos, L. R. (1976). Bilinguals in psychotherapy: Language as an emotional barrier. American Journal of Psychotherapy, 30, 552-560.

Marcos, L. R. (1979). Nonverbal behavior and thought processing. Archives of General Psychiatry, 36, 943-950.

Marcos, L. R. (1980). Bilinguals in Psychotherapy. En M. A. ᄀSimpson (Ed.), Psycholinguistics in Clinical Practice (pp. 91-108). New York, Irvington Publishers, Inc.

Marcos, L. R. \& Alpert, M. (1976). Strategies and risks in psychotherapy with bilingual patients: the phenomenon of language independence. American Journal of Psychiatry, 133, 1275-1278.

Marcos, L. R., Alpert, M., Urcuyo, L., \& Kesselman, M. (1973). The effect of interview language on the evaluation of psychopathology in SpanishAmerican schizophrenic patients. American Journal of Psychiatry, 130(5), $549-553$.

Marcos, L. R., Eisma, J. E., \& Guimon, J. (1977). Bilingualism and sense of self. The American Journal of Psychoanalysis, 37, 285-290.

Pavlenko, A. (2006). En A. Pavlenko (Ed.) Bilingual Minds: emotional experience, expression and representation (pp. 1-33). Clevedon, U.K.: Multilingual Matters.

Perez Foster, R. (1996). The bilingual self: duet in two voices. Psychoanalytic Dialogues, 3, 69-121. 
Laguzzi I Psicodebate, 14(1) I 33-44.

Rozensky, R. H. \& Gomez, M.Y. (1983). Language switching in psychotherapy with bilinguals: two problems, two models and case examples. Psychotherapy: Theory, Research and Practice, 20(2), 152-160.

Schrauf, R. W. (2000). Bilingual autobiographical memory. Experimental studies and clinical cases. Culture and Psychology, 6, 387-417.

Schrauf, R. W. \& Rubin, D. C. (2000). Internal languages of retrieval: the bilingual encoding of memories from the personal past. Memory and Cognition, 28, $616-623$.

Stengel, E. (1939). On learning a new language. International Journal of Psychoanalysis, 20, 471-479.

United Nations Development Programme (2009). Human development report. New York: Palgrave Macmillan 